\title{
Sample-Size Considerations for a Study of Shorebird Nest Survival in the 1002 Area, Arctic National Wildlife Refuge, Alaska
}

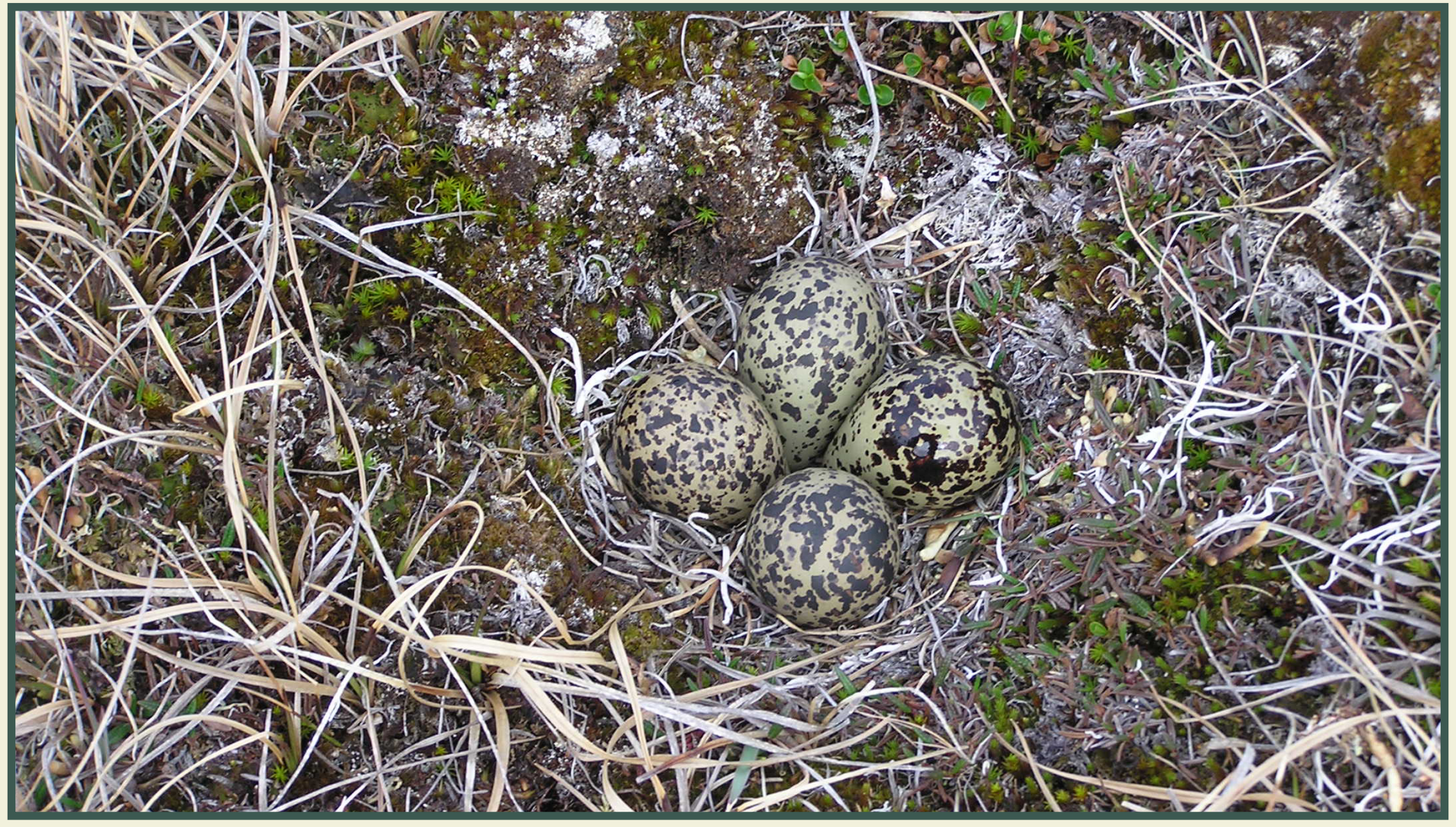

Open-File Report 2020-1066

U.S. Department of the Interior

U.S. Geological Survey 
Cover: Black-bellied plover nest, Prudhoe Bay, Alaska. (Photograph by Emily Weiser, U.S. Geological Survey.) 


\section{Sample-Size Considerations for a Study of Shorebird Nest Survival in the 1002 Area, Arctic National Wildlife Refuge, Alaska}

By Emily L. Weiser

U.S. Geological Survey Wildlife Program

Open-File Report 2020-1066 


\section{U.S. Department of the Interior \\ DAVID L. BERNHARDT, Secretary}

\section{U.S. Geological Survey James F. Reilly II, Director}

U.S. Geological Survey, Reston, Virginia: 2020

For more information on the USGS-the Federal source for science about the Earth, its natural and living resources, natural hazards, and the environment-visit https://www.usgs.gov/ or call 1-888-ASK-USGS (1-888-275-8747).

For an overview of USGS information products, including maps, imagery, and publications, visit https:/store.usgs.gov.

Any use of trade, firm, or product names is for descriptive purposes only and does not imply endorsement by the U.S. Government.

Although this information product, for the most part, is in the public domain, it also may contain copyrighted materials as noted in the text. Permission to reproduce copyrighted items must be secured from the copyright owner.

Suggested citation:

Weiser, E., 2020, Sample-size considerations for a study of shorebird nest survival in the 1002 Area, Arctic National Wildlife Refuge, Alaska: U.S. Geological Survey Open-File Report 2020-1066, 18 p., https://doi.org/10.3133/ofr20201066.

ISSN 2331-1258 (online) 


\section{Contents}



\section{Figures}

1. Map showing nest-monitoring plots in five Alaskan North Slope areas used to inform simulations for this report..

2. Schematic showing data simulation and modeling process used to evaluate how various scenarios, based on sampling design and biological parameters, could be used to identify spatial hotspots of shorebird nest success on the North Slope of Alaska

3. Graphs showing values calculated from previous datasets used to develop the ranges of values included in the simulated scenarios

4. Maps showing expected probability of a nest surviving to hatch for nests of species with biparental or uniparental incubation from the 2019 pilot dataset in the 1002 Area of the Arctic National Wildlife Refuge

5. Graphs showing relative effects of study parameters on the ability to detect hotspots of high nest survival in the 1002 Area

6. Graphs showing proportion of study plots identified as significant hotspots under each scenario, with a total of 68 study plots in the 1002 Area.

7. Graphs showing relationships between study design parameters and the ability to detect hotspots of high nest survival in the 1002 Area.

8. Graphs showing effect of the number of potential nest-days on the ability to detect hotspots under various conditions in the 1002 Area.

9. Examples of how patterns in expected nest survival would be described depending on the number of plots sampled in the 1002 Area

10. Graph showing expected change in mean squared error across spatial units of the study area as the number of study plots increases from 10 to 196 in the 1002 Area. 


\section{Tables}

1. Numbers of nests used to develop parameter estimates from previous datasets at each site, separated by incubation strategy, Alaska's North Slope.

2. Field reference chart for the target number of nests given age of nests found, Alaska's North Slope

\section{Abbreviations}

ASDN Arctic Shorebird Demographics Network

DSR daily survival rate of nests

SD standard deviation

USFWS U.S. Fish and Wildlife Service 


\title{
Sample-Size Considerations for a Study of Shorebird Nest Survival in the 1002 Area, Arctic National Wildlife Refuge, Alaska
}

\author{
By Emily L. Weiser
}

\begin{abstract}
Authorization of lease sales for oil development in the 1002 Area of the Arctic National Wildlife Refuge has highlighted gaps in information about biological communities in the area. The U.S. Fish and Wildlife Service, which is planning a study to evaluate spatial variation in the nest survival of tundra-breeding shorebirds to identify hotspots with high nest survival, sought advice from the U.S. Geological Survey on how to predict which sampling designs might be successful in achieving the study goals. I used previously obtained data on nest survival from Alaska's North Slope to develop a simulation study that explored the statistical consequences of various sampling designs in the context of expected ranges of values of biological parameters. Of all sampling parameters, the number of nests per plot had the strongest influence on the ability to detect hotspots, followed by number of years of monitoring and the age at which nests were found. The benefit of sampling 98 plots instead of 68 was relatively small. Ages of nests cannot be predicted a priori, so I developed a field reference chart to identify when further nestsearching would be useful for a given plot. This simulation study used the best available information, but values of biological parameters will become better defined following subsequent data collection in the 1002 Area. Data from upcoming field seasons could be used to refine this analysis and improve estimation of the expected results of the field study, thus maximizing the likelihood that data from the 1002 Area nest survival study will meet the monitoring goals.
\end{abstract}

\section{Introduction}

Sound knowledge of how the extraction of resources potentially affects Arctic wildlife is needed to inform efforts to minimize or mitigate the impacts of development. Authorization of oil lease sales in the 1002 Area of the Arctic National Wildlife Refuge (Section 20001 of the Tax Cuts and Jobs Act of 2017 (P.L. 115-97)) has highlighted gaps in information about biological communities in the area (Pearce and others, 2018). To address one of these gaps, the U.S. Fish and Wildlife Service (USFWS) is planning a study to evaluate spatial variation in the nest survival of tundra-breeding shorebirds to identify hotspots of productivity where shorebird populations might be disproportionately affected by development (Lanctot and others, 2019). The study will focus on the area northwest of the Marsh Creek Anticline in the 1002 Area (fig. 1). A spatially balanced sampling design has been developed for the study, but questions remain 
around the sampling effort that would provide sufficient data to identify hotspots of high nest survival. Identifying appropriate sample sizes is essential for ensuring that the study has a strong chance of meeting the objectives (Legg and Nagy, 2006; Urquhart, 2012).

In the 1002 Area study, the key elements of shorebird sampling effort include the number of years during which nests are monitored, the number of study plots, and the number of nests per plot. Another consideration is the age at which nests are found, as younger nests can potentially contribute more days of data to the study. While age of discovered nests cannot be predicted a priori, sampling windows can be timed to coincide with appropriate nest ages based on seasonal bird phenology. In the planned USFWS study, logistically feasible sample sizes may include 68-98 plots, 1-4 nests per plot, and 2-3 years of monitoring with the potential to seek further funding for continued monitoring.

The response variable of interest for the 1002 Area shorebird study is the daily survival rate of nests (DSR). Equivalently, survival rates can be expressed as the probability of a nest surviving to hatch, which is calculated as $D S R^{d}$, where $d$ is the length of the species-specific exposure period (egg-laying + incubation) in days. The goals of the study were to identify hotspots (any plot with DSR significantly higher than the overall mean) of nest survival within the study area and to describe spatial patterns in nest survival across the study area. In this report, any plot with DSR significantly higher than the overall mean is considered a hotspot. I also evaluated how sample sizes would influence the spatial resolution of nest survival values extrapolated across the study area.

\section{Methods}

I used available data, as described below, to parameterize a simulation model and to evaluate the statistical consequences of various sampling designs under various biological conditions. The statistical power to detect hotspots will depend on biological parameters such as the mean and variance of DSR, but those values cannot be predicted a priori. I therefore used previous data to define a range of values that I tested in the simulation described below. The previous data came from two previous studies on Alaska's North Slope:

1. 1002 Area pilot study in 2019 (Lanctot and others, 2019). To help develop methods for this study, bird nests were discovered during a single visit to each plot. Eggs from each nest were floated to estimate embryo age (Liebezeit and others, 2007), and a Tinytag temperature logger (Gemini Data Loggers) was placed at each nest to record the incubation temperature. Temperature graphs can be used to infer nest fate, where hatching nests show a period of variable temperature and failed nests show a quick decline in temperature immediately following the nest predation event (R. Lanctot, U.S. Fish and Wildlife Service, personal communication, 22 Nov 2019). Nest fates (hatched or failed) were evaluated by four USFWS reviewers based on the temperature graphs. If reviewers disagreed on the fate of a nest, the fate was recorded as "unknown." In 2019, nests were typically found late in the incubation period based on the ages estimated by flotation, so often only a few days of data were available per nest. Nest survival estimates from this dataset thus may have been biased high, as the monitored nests had already demonstrated high survivorship through most of the incubation period. Except for nestsearching efforts being focused earlier in the nesting season, the Lanctot and others (2019) field methods will be used in future years of the 1002 Area study. 
2. Due to the small samples and expected bias in the 20191002 Area pilot dataset, I also evaluated DSR from data collected by the Arctic Shorebird Demographics Network (ASDN; Lanctot and others, 2016) in 2008-14 at five sites on Alaska's North Slope (fig. 1). Only one ASDN site (Canning Delta) was in the 1002 Area, and ASDN used different study methods (multiple nest-searching visits per plot, frequent checks by observers to evaluate nest status, and visual assessment of evidence at the nest to determine nest fate) than the 1002 Area study used. Estimates of DSR and variance might therefore differ between the ASDN dataset and future years of the 1002 Area study. However, the ASDN dataset provides estimates of DSR, inter-plot variation, and inter-annual variation that are based on robust sample sizes (table 1).

Together, the estimates from the 1002 Area in 2019 and the ASDN sites provided a realistic range of values that I used to inform the data simulations, with multiple scenarios encompassing the expected range of values that may be observed in future years of the 1002 Area study.

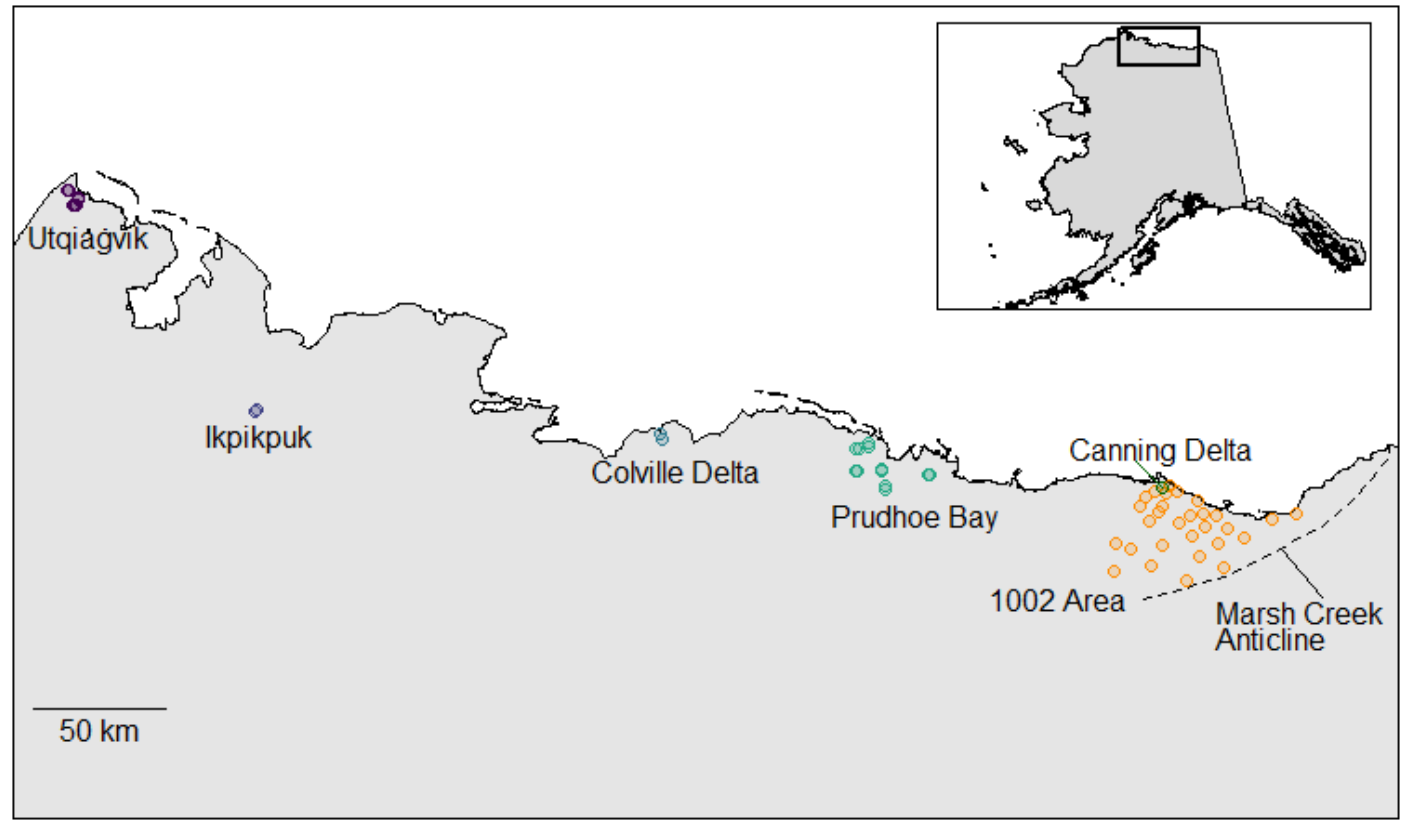

Figure 1. Map showing nest-monitoring plots in five Alaskan North Slope areas used to inform simulations for this report. [Data from the 1002 Area (orange points) were collected as part of a pilot season for this study in 2019, while data from the other sites were collected by the Arctic Shorebird Demographics Network in 2008-14 (Lanctot and others, 2016).]

Table 1. Numbers of nests used to develop parameter estimates from previous datasets at each site on Alaska's North Slope, separated by incubation strategy.

\begin{tabular}{lccc}
\hline \multicolumn{1}{c}{ Site } & Years & Biparental & Uniparental \\
\hline Utqiagivik & $2008-14$ & 816 & 1,167 \\
Ikpikpuk & $2010-14$ & 354 & 192 \\
Colville Delta & $2011-14$ & 457 & 174 \\
Prudhoe & $2010-13$ & 67 & 43 \\
Canning Delta & $2010-14$ & 506 & 774 \\
1002 Area & 2019 & 22 & 33 \\
\hline
\end{tabular}




\section{Parameter Estimates from Previous Data}

To inform the sample size assessment described in the two following subsections of the "Methods," I used the two datasets described at the beginning of the "Methods" section to develop estimates of DSR by site, year, and study plot. I also evaluated the ages at which nests were found, which would affect the potential number of days that a nest could be monitored (that is, until hatching if the nest survived the full incubation period). For each site and year, I used a Bayesian DSR model to estimate the overall mean DSR in each site and year, plot-specific mean for each year (for sites with multiple plots), among-plot variance within each year, and amongyear variance for each plot (at sites with multiple years of data for multiple plots). I evaluated all shorebird species together in one model, as the identity of species to be included in this study is not known. I used the observed ranges of values in the previous datasets to define a set of scenarios that tested multiple values of each parameter, thus encompassing the expected range of possible values that may be encountered in the 1002 Area study. The results of this simulation therefore provide a range of best- and worst-case scenarios that can be considered when determining the final sampling design.

\section{Simulation Study: Detecting Hotspots}

To evaluate the consequences of various sampling designs and biological conditions, I used the parameters estimated from the datasets described at the beginning of the "Methods" section to simulate nest histories. I then analyzed the simulated data and identified which scenarios allowed successful identification of spatial hotspots.

The simulation included scenarios where values were varied for three biological conditions (mean DSR, inter-plot variation in DSR, and inter-annual variation in DSR) and four elements of sampling design (number of years, number of study plots, number of nests [per plot and per year]) and the age at which each nest was found (fig. 2). Simulated values of the sampling parameters were constrained based on expected feasibility for the planned monitoring program. In scenarios with multiple years of data, I assumed that the same plots were monitored each year. If, in contrast, different plots were monitored in each year, the study would not be able to differentiate between spatial and temporal variation. Monitoring the same plots each year would maximize the statistical power to determine whether a given location represented a spatial hotspot.

I assumed that plots and nests were randomly selected in an unbiased, spatially representative way. I also assumed that there was no spatial autocorrelation among plots, as insufficient data were available to examine spatial autocorrelation on a scale relevant for the 1002 Area study. If spatial correlation is present in the system, identifying spatial patterns in nest survival may be easier than indicated by this simulation.

I simulated nest histories for a hypothetical species with a 22-day incubation period, which was the mean for the shorebird species monitored in the 1002 Area in 2019. Assuming that incubation started on the day the fourth egg was laid and that the egg-laying period represented 3 additional days, the total exposure period for each nest was therefore 25 days. Throughout this report, I refer to "nest age" as the number of days after the fourth egg was laid, but nest-monitoring could begin prior to that day if a nest is found during egg-laying. 


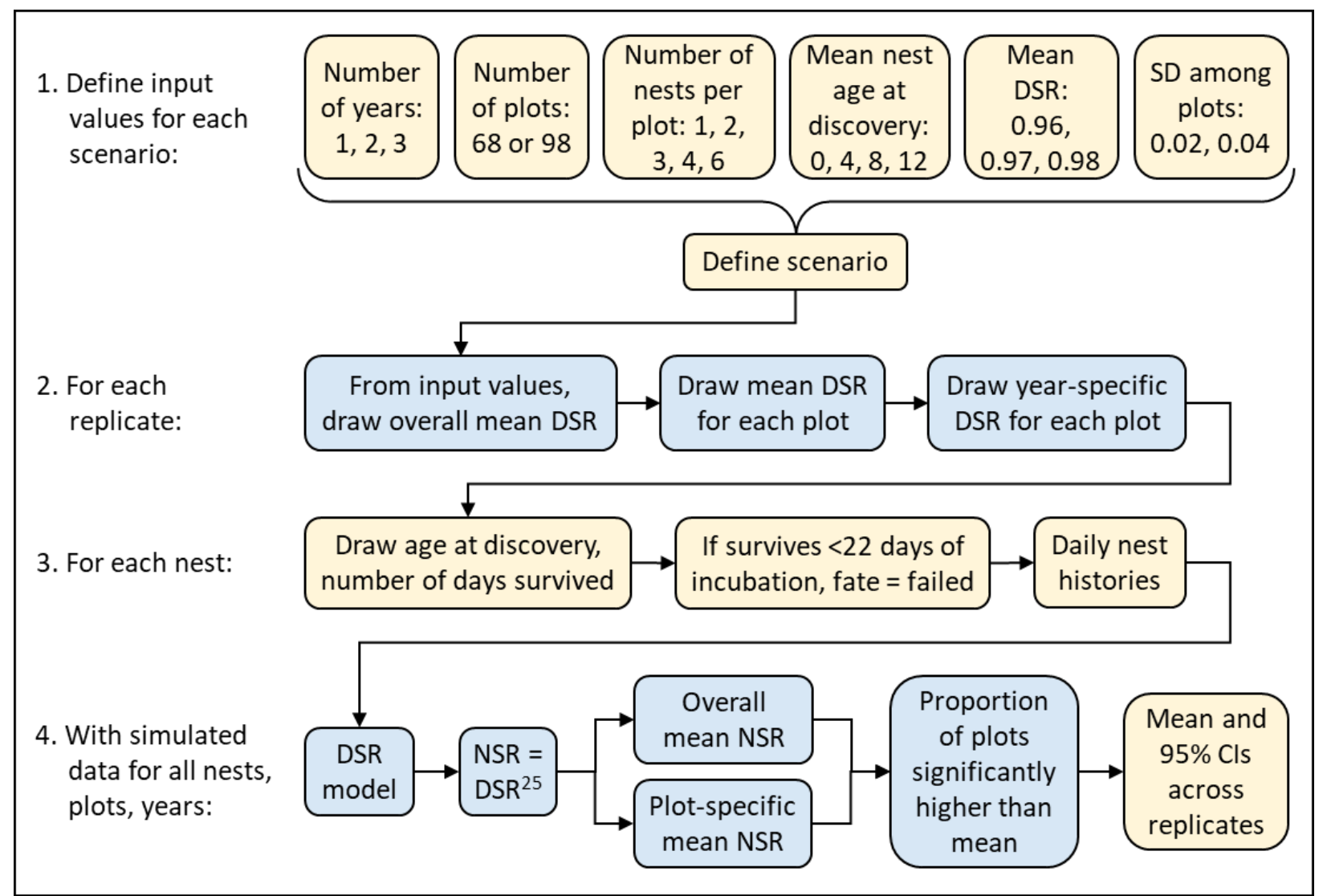

Figure 2. Schematic showing data simulation and modeling process used to evaluate how various scenarios, based on sampling design and biological parameters, could be used to identify spatial hotspots of shorebird nest success on the North Slope of Alaska. [In this report, a "hotspot" is defined as any plot with daily survival significantly higher than the overall mean. A 22-day incubation period was assumed in all cases (total exposure period $=$ egg-laying + incubation $=25$ days). DSR, daily survival rate; NSR, expected probability of nest survival to hatching.]

I simulated each scenario with the following steps in R version 3.6.1 (R Core Team, 2019; script available, on request, from author [eweiser@usgs.gov]; fig. 2):

1. Define each scenario: Based on pilot data and the scenario values, assign the mean age at which nests were found, overall mean DSR, inter-plot standard deviation (SD) of DSR, number of years, number of plots, and number of nests per plot.

2. For each replicate:

a. Randomly draw the mean DSR for each plot based on the overall mean and interplot SD. DSR values were drawn from a beta distribution (constrained to fall between 0 and 1 ).

b. For each plot, randomly draw the year-specific DSR based on the plot mean and inter-annual variation.

3. For each nest:

a. Select the age at discovery from a Poisson distribution based on the scenario mean age at discovery. 
b. Select the number of days after discovery that the nest survives, using a binomial distribution and the plot mean DSR.

c. If the nest survives to the expected hatching date (22 days of incubation), the nest is assumed to have hatched. Otherwise, the nest is marked as "failed." Nest fate is assumed to be known for all nests in this simulation, but nests with unknown fate could be right-censored in the same modeling framework.

d. Populate the daily nest history for each simulated nest.

4. Apply a DSR model in a Bayesian framework (in JAGS version 4.3.0; Plummer, 2003) to the simulated daily nest histories:

a. In the model, DSR depends on an intercept and a random effect of plot, allowing estimation of an overall mean DSR as well as plot-specific means. Uninformative priors are used for each parameter. I did not include other parameters in this model, but inclusion of covariates in the analysis of the field data would be useful to control for any potential systematic differences among plots, such as the age at which nests are found, habitat type, distance to coast, or presence of predator nests or dens.

b. The model generates estimates of overall mean DSR, plot-specific mean DSR, and expected nest survival rate over the full exposure period ( 25 days), where expected probability of nest survival is $\mathrm{DSR}^{25}$. While nest survival depends directly on DSR, nest survival is more intuitive to interpret, so I expressed results of the simulation in terms of nest survival. Patterns would be identical if DSR were displayed instead.

c. For each model, I ran 3,500 iterations for each of 6 chains, of which 500 iterations were discarded as burn-in and adaptation and the remaining iterations were thinned by 3 to reduce autocorrelation, resulting in 1,000 saved iterations used for inference. Those iterations were sufficient to allow model convergence as indicated by model output. The full set of scenarios and replicates took about 3,600 computing hours, which could be completed in about 3 days by running replicates in parallel on a supercomputer (USGS Advanced Research Computing 2020).

5. For each replicate of each scenario, calculate the proportion of study plots that show significantly higher (based on 95 percent confidence interval [95\% CI]) DSR than the overall mean. This indicates the proportion of plots that would be identified as hotspots, which is an index of how likely a given study design would be to identify hotspots. I expected that larger samples would allow detection of smaller differences in NSR and a greater proportion of plots as significantly higher than the overall mean. For each scenario, I calculated the mean and $95 \%$ CI of this metric across the 100 replicates.

After running all scenarios, I compared the relative importance of each input parameter on the ability to detect hotspots by fitting a linear model, which assessed the relationship between the proportion of plots identified as having nest survival significantly higher than the overall mean and the values of all input parameters. I first standardized the input values so that the effect sizes would be directly comparable across the scenario parameters. Larger effect sizes thus indicate parameters with stronger influence on the ability to detect hotspots. 


\section{Simulation Study: Describing Spatial Patterns}

In addition to detecting significant hotspots of nest survival (that is, differences among study plots), evaluating spatial patterns in nest survival (that is, broader regions of high or low survival within the study area) is also a goal of this study. I simulated an illustration of how the number of plots would affect the spatial resolution at which inference could be made, based on hypothetical values of nest survival and hypothetical locations of hotspots and coldspots. I also evaluated how the magnitude of error depended on sample size when predicting nest survival values across the landscape based on a subset of study plots.

First, I randomly selected locations for 196 plots across the study area (double the maximum expected sample size for this study) and randomly assigned a nest survival value to each plot. Then I subsampled those plots to 68 or 98 plots (the target sample sizes for this study), and also a series of scenarios using 10 to 190 plots for illustration purposes. For each subsample, I used inverse distance weighted interpolation to predict nest survival across the study area based on the plots included in the subsample. I mapped the results to provide a visual assessment of how the number of plots may affect the spatial resolution of the inference that could be made about locations of hotspots and coldspots and large-scale patterns in nest survival.

To quantify the accuracy of each scenario, I calculated the mean squared error (MSE) of the interpolated values for each scenario. I assumed the scenario with 196 study plots represented "true" values for each spatial unit (hexagonal grid cell) and subtracted that from the interpolated value from the scenario with each number of plots; then squared those values and took the mean. Smaller values of MSE would indicate more accurate quantification of regional patterns of nest survival. All values and locations of hotspots or coldspots in this exercise were entirely hypothetical, but the resulting maps and MSE provide some idea of how the number of study plots could affect the spatial resolution of a resulting map of patterns in nest survival. Finer spatial resolution and better accuracy of such a map would provide the most detailed spatially explicit information on how development of specific areas could affect shorebird productivity at a population level.

\section{Results and Discussion}

\section{Previous Datasets}

The 2 previous datasets included 55 nests in the 1002 Area in 2019 and 110-1,983 nests at each ASDN site in 2008-14 (table 1). The 1002 Area dataset included three shorebird species with uniparental incubation and four with biparental, while the ASDN dataset included four and eight species, respectively. Nests were generally found between 4 and 8 days of age, except nests of uniparentally incubating species in the 1002 Area were found significantly later in incubation (fig. 3a). Differences in nest age at discovery might reflect differences in parental behavior that affect the likelihood of discovering nests during a rapid search (rather than repeated searches as in ASDN), especially early in incubation. Alternatively, the late timing of the 2019 surveys could have caused the discrepancy between incubation groups; biparentally incubating species typically lay their first nest earlier than uniparental species (Weiser and others, 2018) and may renest following egg depredation (Gates and others, 2013), so the biparental nests in this sample could have largely represented renests (laid after uniparental species initiated their first nests). Searching for nests earlier, relative to shorebird phenology, could address this discrepancy in 
future years. If the discrepancy between incubation groups remains, searchers could focus on biparentally incubating species to target younger nests and thus maximize the potential number of days of data that can be collected for each nest.

In the previous datasets, DSR was generally between 0.95 and 0.99 , but occasionally lower (fig. $3 b$ ). Typically, DSR did not differ between biparentally and uniparentally incubating species at a given site and year (fig. $3 c-g$ ). At sites with data available from multiple study plots, the SD of DSR among plots was variable, from 0.002 (which would result in a $95 \% \mathrm{CI}$ of expected DSR ranging from 0.986 to 0.996 among plots) to 0.042 (95\% CI from 0.848 to 1 ; fig. $3 h$ ). At Utqiagivik, which provided data from multiple plots and years, inter-annual SD was variable among plots (fig. 3i), where the most variable plot showed an SD of 0.02 (year-specific estimates of DSR ranging from 0.921 to 0.994). Study plots in the 1002 Area in 2019 showed spatial variation in DSR but no obvious large-scale patterns or spatial correlation (fig. 4). 







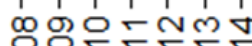

$\infty$ mon

$\infty$ ब)
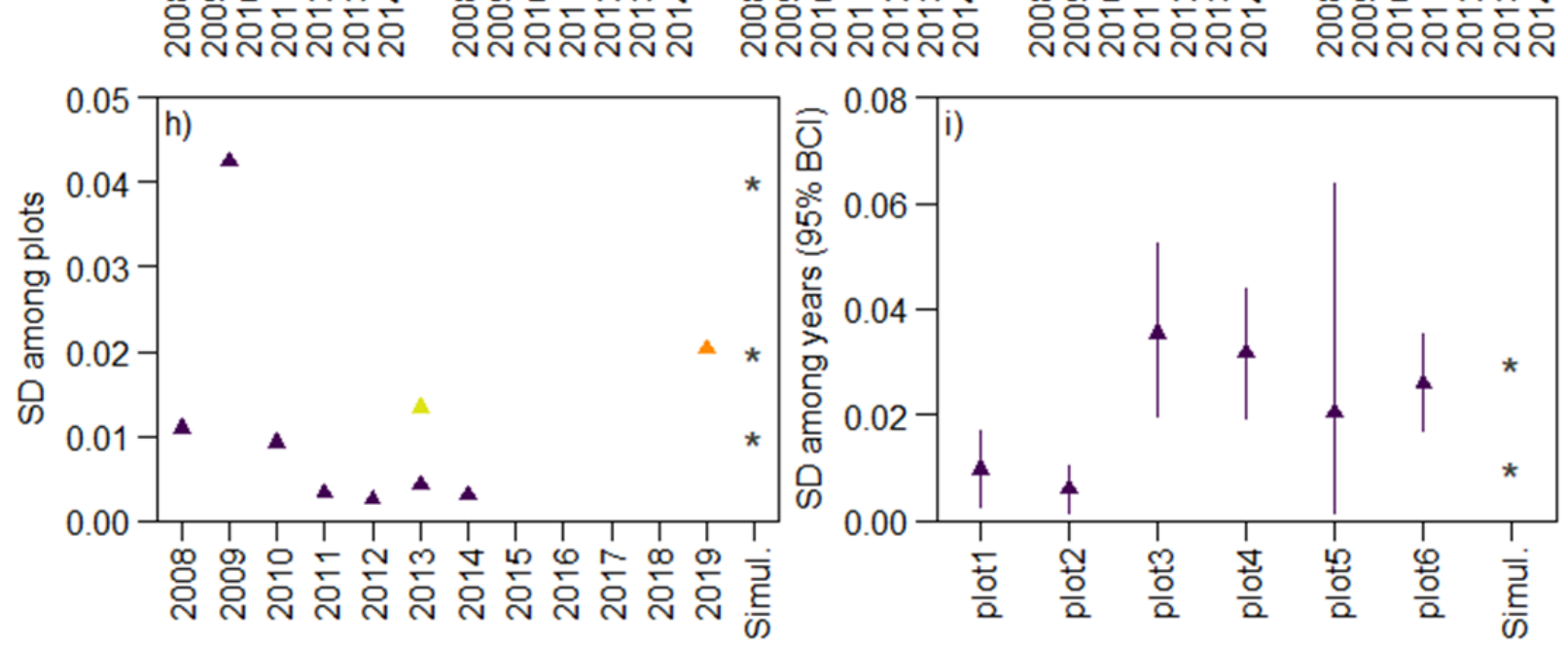

Figure 3. Graphs showing values calculated from previous datasets used to develop the ranges of values included in the simulated scenarios. [(a) age (days of incubation) at discovery; (b) daily survival rate (DSR) by site and year; (c-g) DSR estimates by site, year, and incubation strategy; (h) standard deviation (SD) among plots in DSR; and (i) SD among years for each plot at Utqiagivik. Values used in the simulations are indicated by asterisks and were selected to represent the bulk of the values from the previous datasets.] 

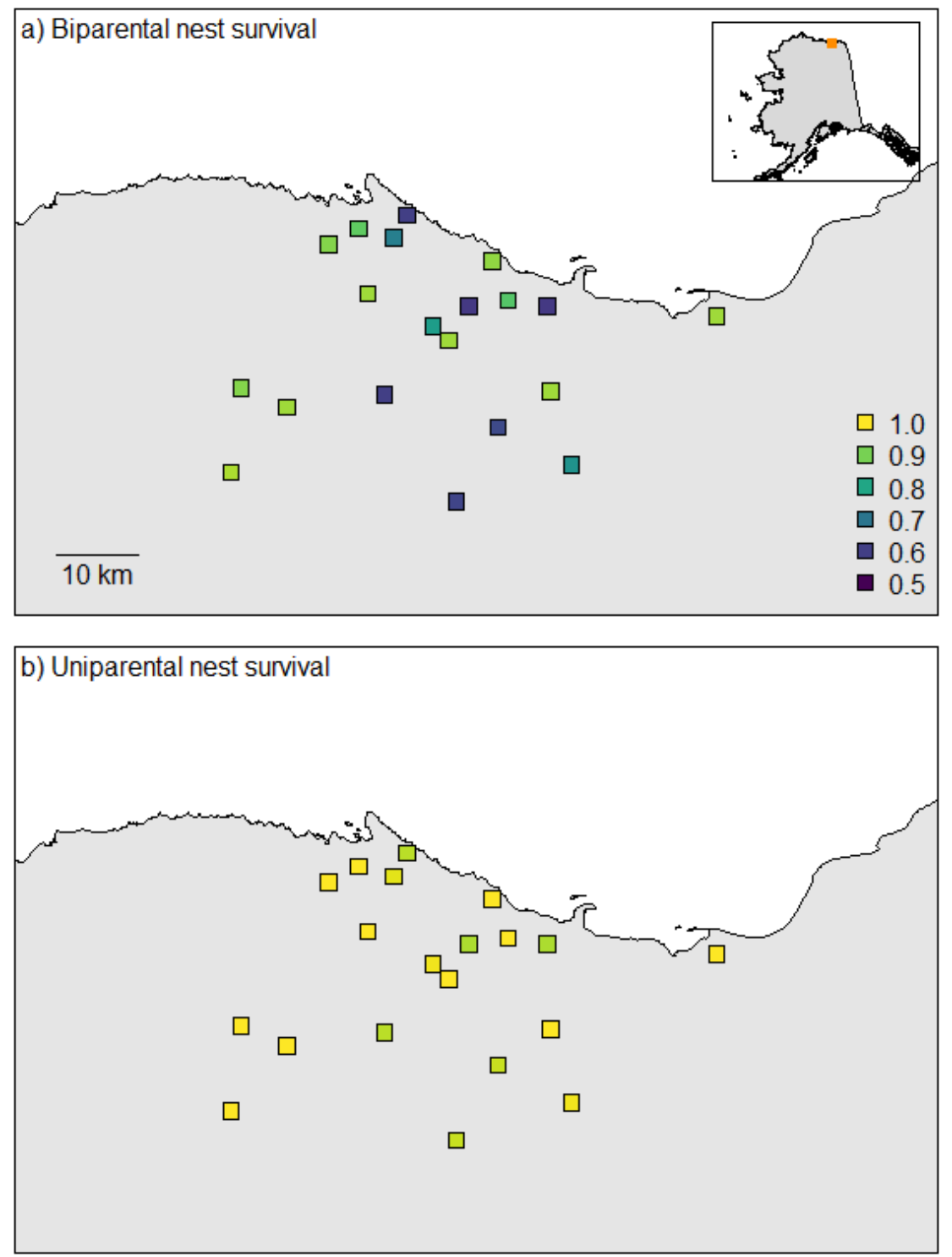

Figure 4. Maps showing expected probability of a nest surviving to hatch (based on observed daily survival rate and a 22-day incubation period) for nests of species with biparental (a) or uniparental (b) incubation from the 2019 pilot dataset in the 1002 Area of the Arctic National Wildlife Refuge. [Inset (a) shows location of the study area in northeastern Alaska. Study plot markers are not drawn to scale.] 


\section{Simulation Results: Detecting Hotspots}

Of the parameters that I varied across simulated scenarios, mean DSR and the inter-plot SD had the strongest effects on the ability to detect hotspots (that is, plots that had significantly higher DSR than the overall mean; fig. 5a). These biological parameters cannot be controlled or predicted a priori, but a sampling design can be chosen that is expected to be successful under a variety of values of these parameters (fig. 6).

Of the sampling parameters that can be determined by the study design, the number of nests per plot and year was most important for the ability to detect hotspots (fig. 5a). Hotspot detection improved quickly as nests increased from 1 to 4 nests per plot; adding additional nests made a progressively smaller difference (fig. 7c), suggesting that a target of 3-4 nests per plot may optimize the return per unit effort. In contrast, the ability to detect hotspots declined more linearly as nest age at discovery increased (fig. 7d), indicating the utility of finding nests as early (young) as possible. Three to 5 years of data were substantially more effective at identifying hotspots than one or two (fig. 7a). There was little difference between monitoring 68 and 98 plots, indicating that either sample size was equally good at detecting significant hotspots (fig. $7 b$ ). However, a larger number of plots would provide better spatial resolution when evaluating landscape-level patterns in nest survival (see next subsection).
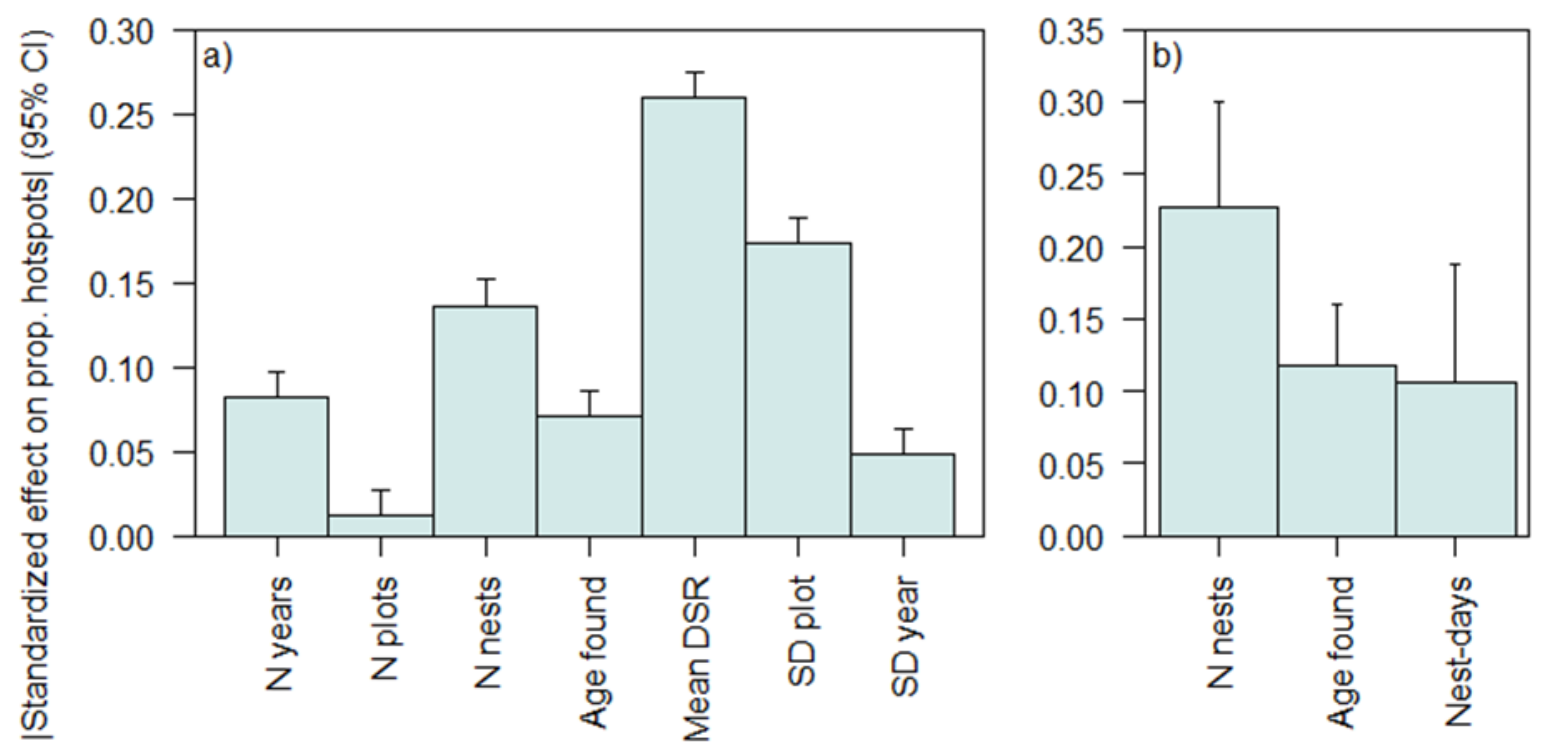

Figure 5. Graphs showing relative effects of study parameters on the ability to detect hotspots of high nest survival in the 1002 Area. [Standardized effect sizes are directly comparable across parameters within panels, but not between panels. (a) Of all parameters tested here, daily survival rate (DSR) and the standard deviation (SD) among plots are most important but not within control of the study design. Of parameters that can be controlled (sample sizes), the number of nests (per plot, per year) is the most influential. (b) Number of nests and age at which nests are found determine the number of potential nestdays per plot and year (without accounting for how long nests actually survive, which cannot be predicted a priori). Age at discovery cannot be predicted well, so number of nest-days could be calculated in the field and additional nest-searching conducted as needed. However, the number of nests is still more influential than the number of nest-days.] 


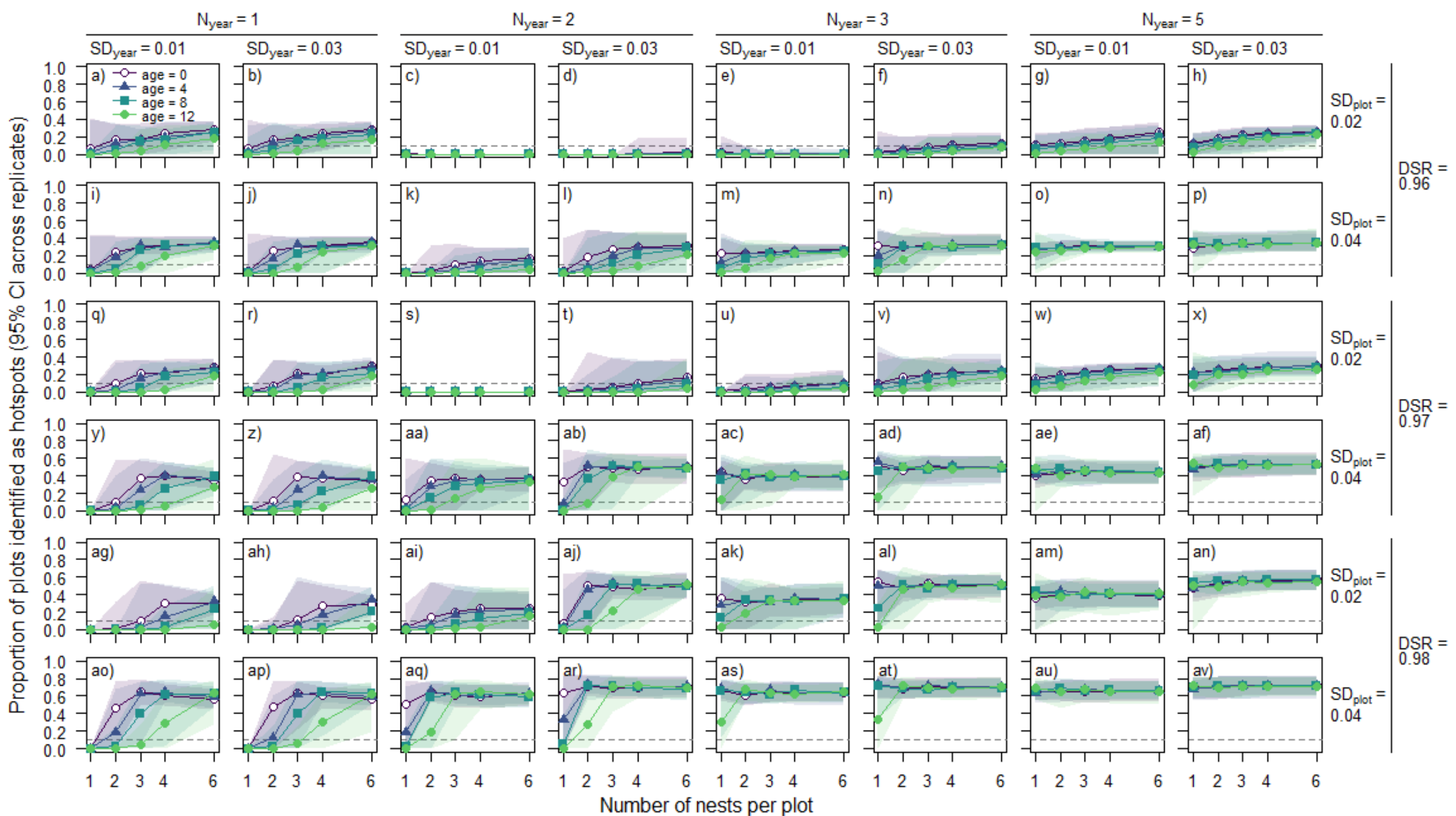

Figure 6. Graphs showing proportion of study plots identified as significant hotspots under each scenario, with a total of 68 study plots in the 1002 Area. [Each scenario included a different value for mean DSR and the SD among plots (rows), the number of years and SD among years (columns), number of nests monitored per plot (x-axis), and mean age (incubation days) at which nests were found (lines). A reference line (dashed gray) is provided at 0.10 to facilitate comparison among panels. Results with 98 plots are nearly identical to those with 68 plots, but this does not consider the potential spatial resolution of inferring spatial variation (see fig. 9).] 
While a target number of nests per plot can be set a priori, and surveys can be timed to coincide with early incubation of most species, the age at which nests are found cannot be directly predicted. Thus, I evaluated whether additional nest-searching would be useful when the target number of nests is found but some of those nests are late in incubation. Together, the age of nests and the number found determine the potential number of nest-days (that is, time remaining between discovery and the expected hatch date) for a given study plot. A larger number of nest-days provides more data than fewer days, but the fundamental sampling unit is still the nest (days are not independent within a nest). Correspondingly, the number of nests was still more influential on the ability to detect hotspots than the number of nest-days (fig. 5b). Still, the number of potential nest-days is a metric that can be calculated in the field as nests are found (based on the estimated ages of nests at discovery).

If nests are found late in incubation, on average, further nest-searching could be useful to provide additional nest-days for a given plot. On average, the return per unit effort leveled off around 50 to 70 nest-days (fig. 7e). The shape of the relationship between nest-days and the ability to detect hotspots depended on other parameters of the scenario, including mean DSR and variance, which cannot be predicted when nests are found (fig. 8). In most cases, 50 nest-days would maximize the ability to detect hotspots; but if variance among plots is low, 70 or more nest-days would be needed to detect hotspots (because low variance results in small differences among plots). Assuming 50 or 70 nest-days are targeted per plot and year, a field reference chart (table 2) can provide guidance for a target number of nests per plot given the age at which nests are found. For example, if the general target is 3 nests per plot but nests at a given plot are found with an average of 10 days remaining before hatch, further nest-searching could be conducted in an effort to monitor 5 nests (to achieve 50 nest-days) or 7 nests (to achieve 70 nest-days) on that plot.

Table 2. Field reference chart for the target number of nests given age of nests found on each plot in the 1002 Area of the Arctic National Wildlife Refuge.

[Mean estimated number of days until hatch: Days remaining until hatch (based on age estimated at discovery and the species-specific incubation period), averaged across nests already found on the plot in a given year. Target number of nests: Per plot and year]

\begin{tabular}{ccc}
\hline $\begin{array}{c}\text { Mean estimated } \\
\text { number of days until } \\
\text { hatch }\end{array}$ & \multicolumn{2}{c}{ Target number of nests } \\
\cline { 2 - 3 } $21-22$ & For 50 nest-days & For 70 nest-days \\
$16-20$ & 3 & 3 \\
15 & 3 & 4 \\
$13-14$ & 3 & 5 \\
12 & 4 & 5 \\
11 & 4 & 6 \\
10 & 5 & 6 \\
9 & 5 & 7 \\
8 & 6 & 8 \\
7 & 6 & 9 \\
\hline
\end{tabular}




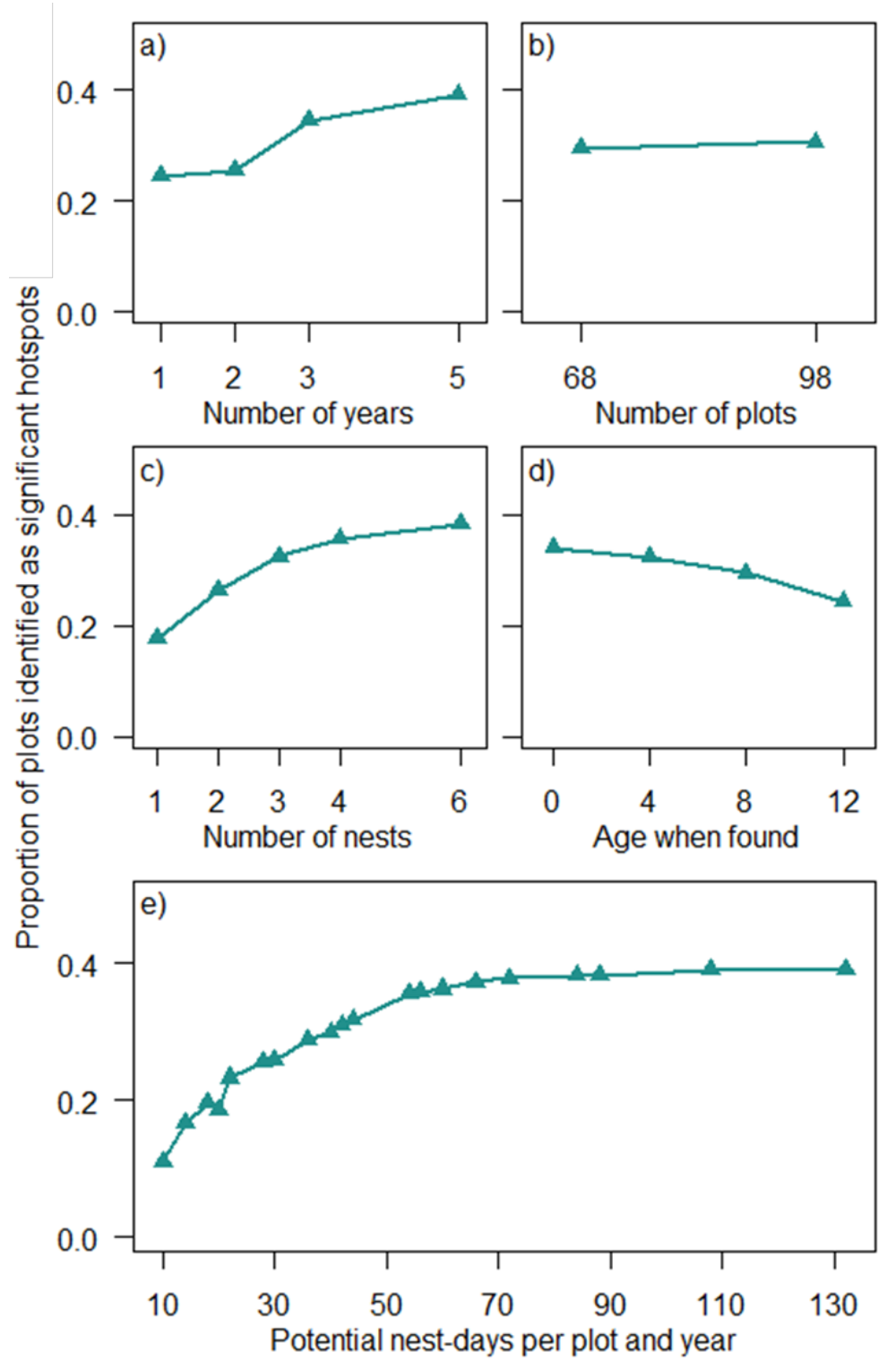

Figure 7. Graphs showing relationships between study design parameters and the ability to detect hotspots of high nest survival in the 1002 Area. [In this report, a "hotspot" is defined as any plot with daily survival significantly higher than the overall mean. The specific consequences of a given value of each design parameter (that is, the exact proportion of plots identified as hotspots) will depend on the values of other parameters, but the curves shown here indicate how, on average, the ability to detect hotspots will be affected by the indicated design parameter. See also fig. 8 for how the shape of the relationship in $(e)$ depends on other parameters.] 

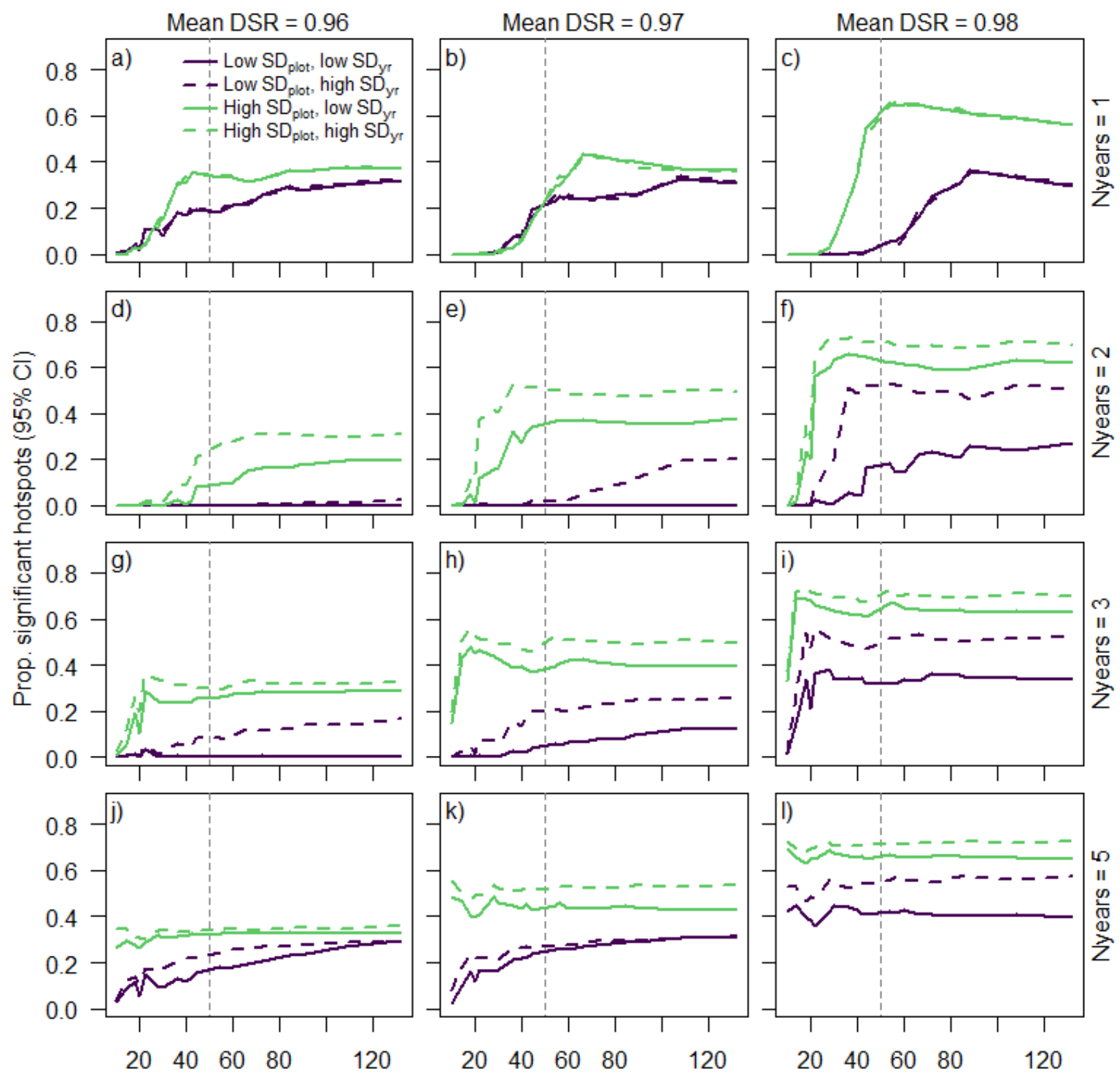

Potential nest-days per plot and year

Figure 8. Graphs showing effect of the number of potential nest-days on the ability to detect hotspots under various conditions in the 1002 Area. ["Potential nest-days" is the time remaining in the expected incubation period after the nest was found, summed across nests in that plot for each year. A reference line is shown at 50 nest-days on each panel. The point at which the ability to detect hotspots levels off varies depending on the other parameters. This figure assumes 68 plots are monitored, but values would be nearly identical for 98 plots. Nyears, number of years; SD, standard deviation] 


\section{Simulation Results: Spatial Resolution and Accuracy}

As expected, spatial resolution of patterns in nest survival was finer when larger numbers of plots were sampled (fig. 9). Similarly, the error between the "true" simulated values and the values interpolated from a subset of study plots declined as the number of plots increased. Error was reduced quickly as the number of plots increased to 60 and more gradually with larger sample sizes (fig. 10). While sampling 98 plots instead of 68 improved the spatial resolution of interpolated nest survival, the larger sample size did not improve the ability to detect hotspots (figs. 5 and 7) and produced a relatively small improvement in MSE (fig. 10).

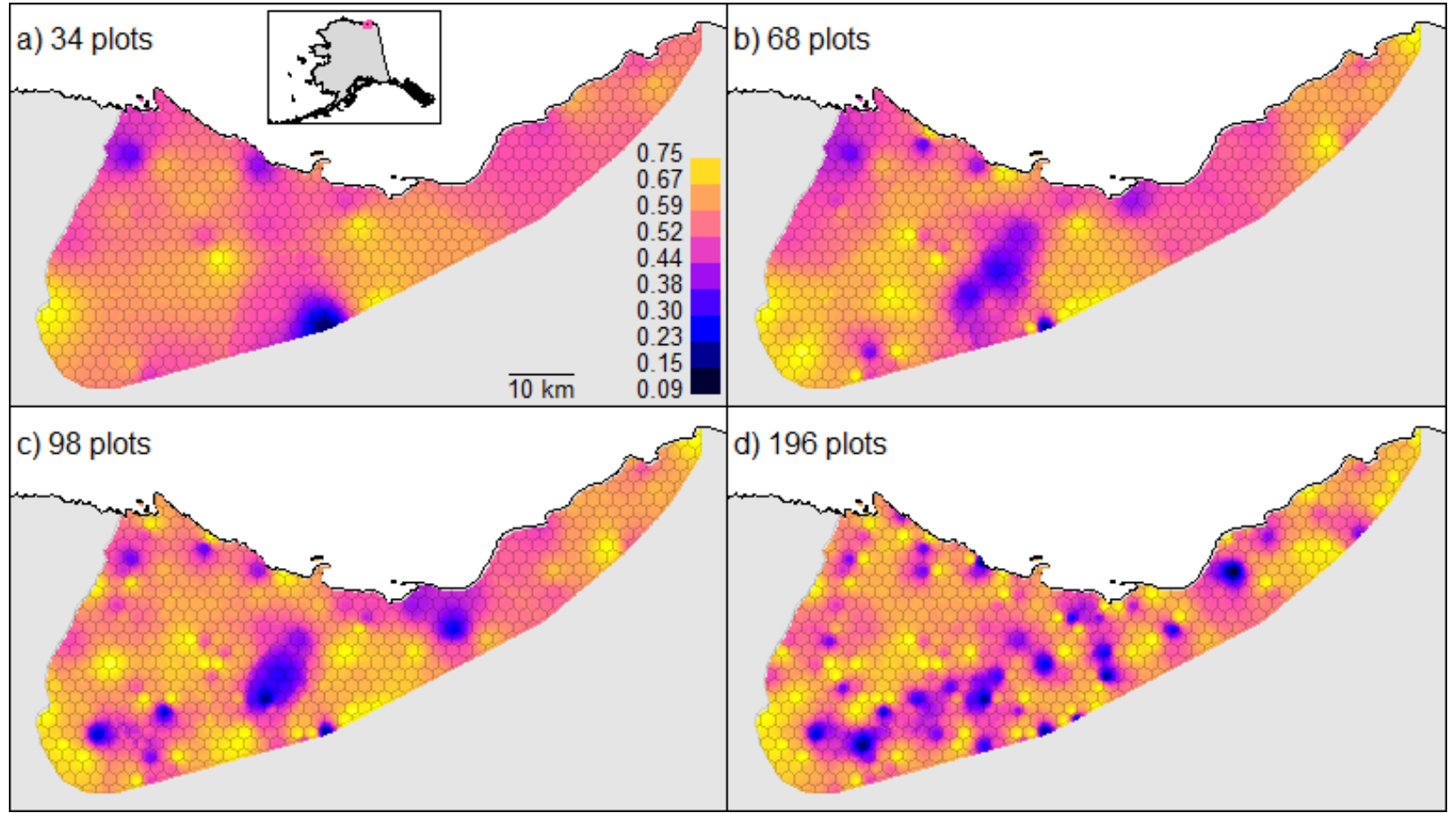

Figure 9. Examples of how patterns in expected nest survival would be described depending on the number of plots sampled in the 1002 Area. [This exercise used random values and patterns, so the locations of hotspots and coldspots shown here are not meaningful. However, a visual comparison across the panels indicates the relative resolution of spatial predictions that could be gained from the indicated number of study plots, as well as the potential to miss hotspots or coldspots within the study area. Inset (a) shows location of the study area in northeastern Alaska.] 




Figure 10. Graph showing expected change in mean squared error across spatial units of the study area (fig. 9) as the number of study plots increases from 10 to 196 in the 1002 Area. [Vertical dashed lines are shown for reference at 68 and 98 plots.]

\section{Conclusions}

Of sampling parameters, the number of nests per plot had the strongest influence on the ability to detect hotspots (three or four nests per plot per year could maximize the return per unit effort), followed by number of years of monitoring (more years improved hotspot detection) and age at which nests were found (nests found earlier in incubation provided more power to detect hotspots).

The benefit of sampling 98 plots instead of 68 may be relatively small, as the ability to detect significant hotspots did not differ and MSE changed relatively little between the two scenarios. However, more plots would provide better spatial resolution for predicting nest survival across the study area and would reduce the risk of missing hotspots that could be important to identify in the context of 1002 Area land management and potential future development. In either case, the simulations assumed that the same plots would be monitored across all years of the study.

Targets for the number of nests per plot can be developed prior to the field season. While in the field, a reference chart for the target number of nests given the average age of nests already found can be used to identify when further nest-searching would be useful for a given plot.

All of the results presented here depend on hypothetical scenarios that were informed by ranges of input values that have been observed in previous datasets, including data from outside the 1002 Area. While these values provide the best estimates that would be relevant to this study, the values used here may not accurately describe the data that will be collected for this study. The analyses presented here could be updated with additional data from the 1002 Area collected in summer 2020 to improve estimation of the expected results of the study, given parameters observed in the study area to date, and thus maximize the likelihood that data from this study will be sufficient to meet the monitoring goals. 


\section{Acknowledgments}

I thank R. Lanctot, S. Saalfeld, and C. Latty (U.S. Fish and Wildlife Service) and S. Schulte and S. Brown (Manomet) for providing unpublished data from 2019 and for discussion that improved the relevance of this analysis.

\section{References Cited}

Gates, H.R., Lanctot, R.B., Powell, A.N., 2013, High renesting rates in Arctic-breeding Dunlin (Calidris alpina) — A clutch-removal experiment: Auk, v. 130, 372-380.

Lanctot, R., Latty, C., Sowl, K., Muir, J., Hoepfner, S., Saalfeld, S., 2019, Monitoring avian nest survival on the Arctic National Wildlife Refuge, in Annual Summary Compilation-New or ongoing studies of Alaska shorebirds: Alaska Shorebird Group, p. 21-22.

Lanctot, R.B., Brown, S.C., Sandercock, B.K., 2016, Data from-Arctic Shorebird Demographics Network: National Science Foundation Arctic Data Center, https://arcticdata.io/catalog/view/doi:10.18739/A28P5V92S.

Legg, C.J., and Nagy, L., 2006, Why most conservation monitoring is, but need not be, a waste of time: Journal of Environmental Management, v. 78, p. 194-199.

Liebezeit, J.R., Smith, P.A., Lanctot, R.B., Schekkerman, H., Kendall, S.J., Tracy, D.M., Rodrigues, R.J., Meltofte, H., Robinson, J.A., Gratto-Trevor, C., McCaffery, B.J., Morse, J., Zack, S.W., Tulp, I., Kendall, S.J., Tracy, D.M., Rodrigues, R.J., Meltofte, H., Robinson, J.A., Gratto-Trevor, C., McCaffery, B.J., Morse, J., and Zack, S.W., 2007, Assessing the development of shorebird eggs using the flotation method-Species-specific and generalized regression models: Condor 109, p. 32-47.

Pearce, J. M., Flint, P. L., Atwood, T. C., Douglas, D. C., Adams, L. G., Johnson, H. E., and others, 2018, Summary of wildlife-related research on the coastal plain of the Arctic National Wildlife Refuge, Alaska, 2002-17: U.S. Geological Survey Open-File Report 2018-1003, doi:10.3133/ofr20181003.

Plummer, M., 2003, JAGS_-Program for analysis of Bayesian graphical models using Gibbs sampling, in Hornik, K., Leisch, F., Zeileis, A. (eds.), Proceedings of the 3rd International Workshop on Distributed Statistical Computing (DSC 2003).

R Core Team, 2019, R-A language and environment for statistical computing: Vienna, Austria, R Foundation for Statistical Computing, version 3.6.1, https://www.R-project.org/.

Urquhart, N.S., 2012, The role of monitoring design in detecting trend in long-term ecological monitoring studies, in Gitzen, R.A., Millspaugh, J.J., Cooper, A.B., Licht, D.S. (eds.), Design and analysis of long-term ecological monitoring studies: Cambridge University Press, United Kingdom, p. 151-173.

U.S. Geological Survey, 2020, Advanced research computing-2020 USGS Yeti supercomputer: U.S. Geological Survey, web, https://doi.org/10.5066/F7D798MJ.

Weiser, E.L., Brown, S.C., Lanctot, R.B., Gates, H.R., Abraham, K.F., Bentzen, R.L., and others, 2018, Life-history tradeoffs revealed by seasonal declines in reproductive traits of Arcticbreeding shorebirds: Journal of Avian Biology, v. 49, jav01531. 
Publishing support provided by the U.S. Geological Survey Science Publishing Network, Tacoma Publishing Service Center

For more information concerning the research in this report, contact the Director, Alaska Science Center

U.S. Geological Survey

4210 University Drive

Anchorage, Alaska 99508

https://www.usgs.gov/centers/asc/ 


\section{$\frac{\mathbb{3}}{3}$}

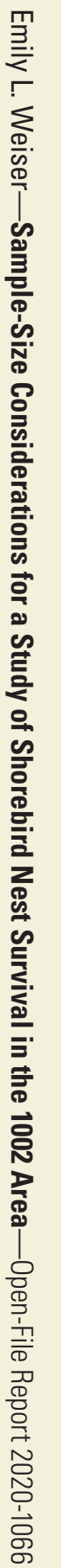

